\title{
Some Properties of Hyperbolic Networks
}

\author{
Christophe Papazian ${ }^{1}$ and Eric Rémila ${ }^{1,2}$ \\ 1 Laboratoire de l'Informatique du Parallélisme CNRS UMR 5668 \\ Ecole Normale Supérieure de Lyon \\ 46 Allée d'Italie, 69364 Lyon Cedex 07, France \\ Christophe.Papazian@ens-lyon.fr \\ 2 GRIMA, IUT Roanne, Université J. Monnet \\ 20 Avenue de Paris, 42334 Roanne Cedex, France \\ Eric.Remila@ens-lyon.fr
}

\begin{abstract}
Many mathematical results exist about continuous topological surfaces of negative curvature. We give here some properties of discrete regular tessellations on such objects and explain a characterization of discrete geodesics and areas that shows how such hyperbolic networks can be seen as intermediary structures between Euclidean infinite tessellations (like square grid) and regular infinite trees.

We do not use some possible group structures of this networks (Cayley graphs) but only geometrical arguments in our constructive proofs. Hence we can see that there are few geodesics in hyperbolic networks and that large areas have very unsmooth borders.
\end{abstract}

\section{Introduction}

A lot of interesting and deep results (see for example [1], [4] and the proceedings of the previous DGCI conferences) have been found about the topology of the three classical regulars tessellations of the Euclidean plane, (with squares, equilateral triangles or hexagons). These tessellations are seen as discrete versions of the plane, they are often called Euclidean discrete planes. In computer imagery, each cell of such a tessellation is viewed as a pixel of a flat screen.

At the opposite, there are very few results about regular tessellations of hyperbolic planes, even though these tessellations also are very interesting : to be more explicit, if a screen were a part of an hyperboloid, then it can canonically be divided in "hyperbolic pixels", which are cells of a tessellation of a hyperbolic plane.

In this paper, we are focused on geodesic lines of these tessellations. We have two main results, which show large structural differences between hyperbolic discrete planes and the Euclidean discrete plane. The proofs of these results are based on precise isoperimetric inequalities in hyperbolic planes (we also give a general isoperimetric inequality).

Our first result describes the set of geodesic lines between two fixed points. We prove that this set is "thin": precisely, there exists a positive constant $c$ such 
that if $a_{1}, a_{2}, a^{\prime}$ and $a^{\prime \prime}$ are points such that $d\left(a_{1}, a^{\prime}\right)+d\left(a^{\prime}, a_{2}\right)=d\left(a_{1}, a^{\prime \prime}\right)+$ $d\left(a^{\prime \prime}, a_{2}\right)=d\left(a_{1}, a_{2}\right)$ and $d\left(a_{1}, a^{\prime}\right)=d\left(a_{1}, a^{\prime \prime}\right)$, then $d\left(a^{\prime}, a^{\prime \prime}\right) \leq c$.

Our second result proves that for each 3-tuple $\left(a_{1}, a_{2}, a_{3}\right)$ of points of the hyperbolic plane, the triangle $\left(a_{1}, a_{2}, a_{3}\right)$ is (nearly) empty. This result can be seen as a discrete version of the classical result about triangles of a continuous hyperbolic plane [3].

\section{Definitions}

A graph is a set $G=(V, E)$ with $V$ a set of vertices, and $E$ a symmetric subset of $V^{2}$, whose elements are called "edges".

$a, b \in V$ are called neighbors if $(a, b) \in E$.

$a \in V$ has a degree $n \in \mathbb{N}$ if $a$ has $n$ differents neighbors.

A path $c$ of a graph is a sequence of vertices, $\left(v_{1}, v_{2}, \ldots, v_{n}\right)$, such that for all $i, v_{i}$ and $v_{i+1}$ are neighbors.

A planar graph is a graph with a planar representation (no crossing edges).

A cycle is a path such that $v_{1}=v_{n}$. Given a planar representation of the graph, a cycle $c$ cuts the set of vertices into three subsets : Int $(c)$, the finite set of vertices inside the cycle, $c$, the vertices of the cycle, and $\operatorname{Ext}(c)$, the set of vertices outside the cycle.

A cell is a cycle with interior void and no edge inside.

Definition 1. A network $\Gamma(k, d)$ is the unique planar graph, with every cell of size $k$ and with every vertex of degree $d$ whose planar representation does not contain an accumulation point.

So, $\Gamma(4,4)$ is the infinite grid isomorphic to $\mathbb{Z}^{2}$.

Definition 2. A geodesic $x y$ is a path from the vertex $x$ to the vertex $y$ such that there is no strictly shorter path.

For all $\Gamma(k, d)$, we note $\gamma=d k-2 d-2 k=(k-2)(d-2)-4$ the curvature of the network. If $\gamma \geq 0$ then the network is infinite. If $\gamma=0$, then the network is called Euclidean, if $\gamma \geq 1$ then the network is hyperbolic. Now, we will only consider hyperbolic networks $\Gamma(k, d)$ (see [2] for details).

For a path, or a set of vertices $c$, we note $|c|$ the number of vertices it contains.

\section{Fundamental Properties}

First, some basic geometric properties, from Euler Formula :

Lemma 1. A completely triangulated planar graph with $n$ vertices on the border and $m$ interior vertices is composed of $n+2 m-2$ triangles (cells of size 3), and $2 n+3 m-3$ edges.

Then, from the previous Lemma, we can deduce the more general lemma : 
Lemma 2. A planar graph whose every cell is of size $k$ (with every vertex into some cell, no vertex of degree 1$)$, with $n$ vertices on the border and $m$ interior vertices is composed of $\frac{(n+2 m-2)}{(k-2)}$ cells and $\frac{(n+2 m-2)}{(k-2)} \times \frac{k}{2}+\frac{n}{2}=n+m-$ $1+\frac{n+2 m-2}{k-2}$ edges.

Proof. We just triangulate cells : there are $k-2$ triangles in a cell of size $k$, and the triangulation needs $k-3$ additional edges for each cell.

Theorem 1 (Isoperimetric inequality). For a cycle $c$ of a $\Gamma(k, d)$, we have :

$$
|\operatorname{Int}(c)| \leq \frac{k}{(k-1) \gamma} \cdot(|c|-2(k-1))
$$

Proof. We have to consider the planar subgraph $G=(V, E)$ of a network $\Gamma(k, d)$ such that $V=c \cup \operatorname{Int}(c)$ and $E$ is the set of all edges inside $c$ or in $c$. So $G$ is composed of cells of size $k$.

$G$ has at least 2 leaving arc for each border vertex plus one every $k-1$ border vertices (if we consider the border larger than a cell) to close the cells and $d$ leaving edges for each interior vertex. So we can deduce the following inequality (using lemma 2 , and $m=|\operatorname{Int}(c)|$ et $n=|c|$ ) :

$$
n+m-1+\frac{(n+2 m-2)}{(k-2)} \geq \frac{m d}{2}+n+\frac{1}{2} \cdot\left\lceil\frac{n}{k-1}\right\rceil
$$

As the border is a cycle, we have to obviously round up the fractional part (if we note $p$ the number of leaving edges, it verifies $p(k-1) \geq n$ so $p \geq \frac{n}{k-1}$ ).

$$
\begin{gathered}
\frac{(n+2 m-2)}{(k-2)}-1 \geq \frac{m(d-2)}{2}+\frac{n}{2 \cdot(k-1)} \\
(n+2 m-2)-(k-2) \geq \frac{m(\gamma+4)}{2}+\frac{n}{2}-\frac{n}{2(k-1)} \\
n-2 k+\frac{n}{k-1} \geq m \gamma \\
k(n-2(k-1)) \geq m \gamma(k-1)
\end{gathered}
$$

Hence, we obtain a linear upper bound of area by perimeter, that is a specific property of hyperbolic networks, whereas area is quadratically majored by perimeter in Euclidean networks. We remark that the curvature of the plane naturally comes into the inequality. Areas are as little as curvature is great: $d$ alone doesn't make any differences, and $k$ is of little importance in the factor $\frac{k-1}{k}$ and in the constant $2(k-1)$. 

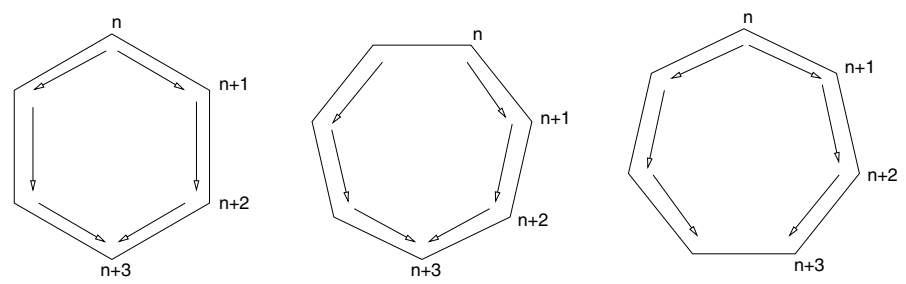

Fig. 1. Orientation of cells for $k=6$ and $k=7$

Lemma 3. Every cell is correctly oriented according to the distance from a given vertex. If $k$ is even, there is one closest point and one farvest point, if $k$ is odd, there is one closest point or one farvest point and "a transversal edge" : one edge that links two edges at the same distance (see figure 1).

Proof. By induction on the building of the network from the given vertex (figure 6).

So, for any vertex and any cell (with $k$ even), there is only one vertex on the cell that minimize the distance with the given vertex. Informally, this property could be seen as a topological uniformity of the metric space. We must note that this property is still true on Euclidean networks, but is false on finite network (when $\gamma<0$ ).

\section{Characterization of Geodesics}

Given two points $x$ and $y$ of a network $\Gamma(k, d)$, with distance $\lambda$ from each other, we consider the subgraph formed by all vertices on any geodesic $x y$ and the edges of this geodesic (There are no transversal edges).

We must note that, for any point $z$ of a geodesic $x y$, the path composed by two arbitrary geodesics $x z$ and $z y$ is a geodesic $x y$.

Lemma 4. The graph of geodesics $x y$ is a planar subgraph delimited by two exterior geodesics.

Proof. (By contradiction) We consider both half-borders of the subgraph that links $x$ to $y$ (it is possible because $x$ and $y$ are compulsory on the border, due to the fact that we consider only infinite surfaces). If one of this path is not a geodesic, then there exists an oriented arc $(a, b)$ of this path that is a part of a geodesic $y x$ and not a $x y$ one. But, as $a$ and $b$ are on the border, we note that, due to planarity (see figure 2), this geodesic $y x$ must cross itself : it must pass trough the same point twice. It is obviously not possible for a geodesic.

The graph of geodesics is planar (as a subgraph of a planar graph), and due to lemma 3 , the graph of geodesics is only composed of cell of size $k$ if $k$ is even, and $2 k-2$ if $k$ is odd. 


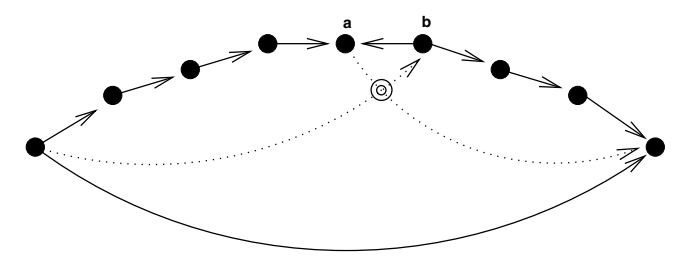

Fig. 2. The impossible geodesic

Theorem 2 (Theorem of neighbor geodesics). Given two vertices $x$ and $y$ of a network $\Gamma(k, d)$, and two geodesics xy (that we note $u$ and $v$ ). Then, Int $\left(u v^{-1}\right)$ is empty.

Proof. We first consider $\mathrm{k}$ as an even number. We use the same method as for Theorem 1 of isoperimetric inequality. We consider that the cycle (border of the area) is $c=u v^{-1}$, and we define the subgraph $G$ of border $c$. We suppose (with any loss of generality) that $u$ and $v$ are disjoint (they share no vertex).

$G$ has at least 2 leaving edges for each border vertex and $d$ leaving edges for each interior vertex. Additionally, if we consider $k-1$ consecutive vertices on $u$ (or $v$ ), we remark the necessary presence of at least 2 supplementary leaving edges, because we have to respect the fact that $u$ (and $v$ ) is a geodesic and that cells are of size $k$, as we can see from figure $3: u$ can follow the boundary of a cell during at most the half of the perimeter.

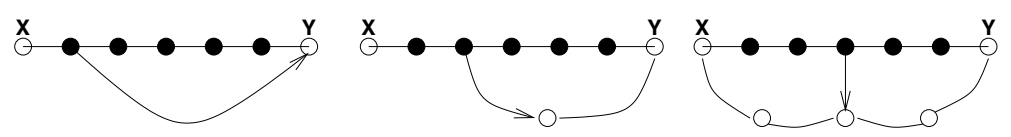

Fig. 3. Impossible to add only one leaving arc on $k-1$ consecutive vertices with the necessity of having a geodesic (between $\mathrm{X}$ and $\mathrm{Y}$ for $k=6$ here)

So, we can deduce the following formula (using Lemma 2 and $m=\operatorname{Int}(c)$ and $n=|c|$ ). We note that here, the fractional part has to be rounded down, as the proposition "at least 2 leaving arc for k-1 vertices" is true on two paths $u$ and $v$ (but not on the entire cycle). (We remark that $\lfloor\nu / \omega\rfloor \geq \frac{\nu+1}{\omega}-1$ ):

$$
\frac{(n+2 m-2)}{(k-2)} \times \frac{k}{2}+\frac{n}{2} \geq \frac{m d}{2}+n+\frac{n+1}{k-1}-1
$$

that we can simplify (as for Theorem 1) :

$$
2 n-6 k+6 \geq m \gamma(k-1)
$$


Now, we will prove that $m$ is not large compare to $n / 2$. Using that fact, we will split our problem $P_{m}$ into two smaller ones $P_{m^{\prime}}^{\prime}$ and $P_{m^{\prime \prime}}^{\prime \prime}$ with the property $m=m^{\prime}+m^{\prime \prime}$. To solve the entire problem, we will split every subproblem until they are trivial (the graph of geodesics is reduced to a single cell where $m=0$ ) which implies that $m=0$ for the initial problem.

The trivial cases are $n=k$ and $n=2 k-2$ where obviously $m=0$.

If we consider $m>n / 2-k$, we can deduce by using the last inequality :

$$
2 n-6 k+6 \geq m \gamma(k-1)>\left(\frac{n}{2}-k\right) \gamma(k-1)
$$

that we can simplify into :

$$
(n-2 k)(\gamma(k-1)-4)<12-4 k
$$

As $k \geq 4$, we remark that $(\gamma(k-1)-4)>0$, so we have $n<2 k$ for $m>0$ ! So $m \leq n / 2-k$.

In every graph of geodesics of border $c=u v^{-1}$, with $u$ and $v$ disjoint, $m \leq$ $n / 2-k$ means that for some $\delta$ such that $k / 2 \leq \delta \leq d(x, y)-k / 2$, there is no interior vertex $\mu$ such that $d(x, \mu)=\delta$ (because there are $n / 2-k+1$ such different $\delta$, and not as many interior points). So there are only two vertices $a$ and $b$ with distance $\delta$ from $x$, one on $u$ and the other on $v$. Moreover, there is no edge $a b$ (it would be a transversal edge !) and no edge between $a$ and $b$ because such an edge would link a vertex with distance $d<\delta$ to another vertex with distance $d>\delta$. Then, $a$ et $b$ are on a cell of the graph of geodesics : There is a cell $F$ which contains $a$ and $b$ (see figure 4 . As $k / 2 \leq \delta, F$ does not contain $x$, and as $\delta \leq d(x, y)-k / 2, \mathrm{~F}$ does not contain $y$.

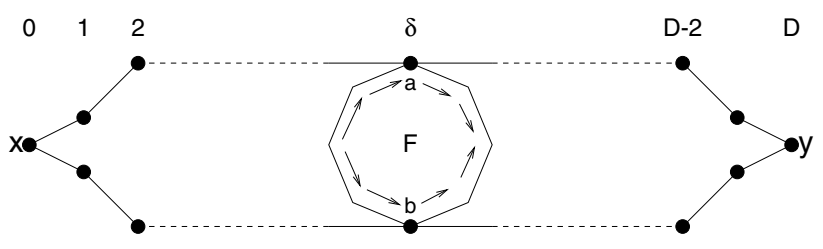

Fig. 4. A cell in the middle

We can now cut this problem into two strictly smaller ones, by using lemma 3 : one that consider the graph of geodesics $x F_{x}$ where $F_{x}$ is the vertex of $F$ farvest from $x$ and the graph of geodesics $y F_{y}$.

We must remark that any geodesic $x y$ can be obtained by concatenation of two geodesics $x a$ and $a y$, or $x b$ and $b y$, due to the fact that $a$ and $b$ are the only vertices $v$ that verify $\{d(x, v)=\delta ; d(x, y)=d(x, v)+d(v, y)\}$. This is still true for any geodesic $x F_{x}$ or $y F_{y}$. So the graph of geodesics $x y$ is actually "split" into two smaller ones $x F_{x}$ and $y F_{y}$. 

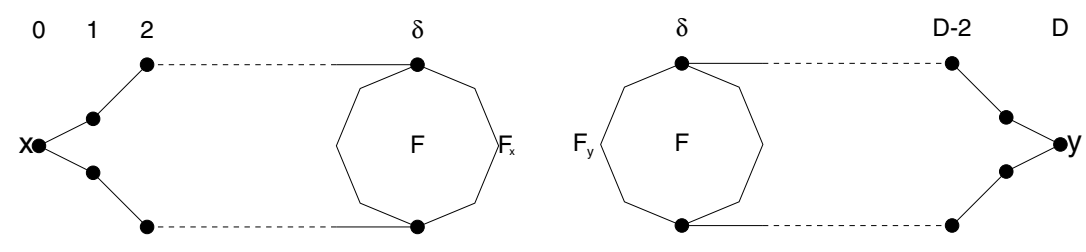

Fig. 5. Two smaller problems

The interior vertices of the initial problem will be interior vertices of exactly one of the two smaller problems, that we can cut until they are reduce to a very small trivial problem ( $c$ is one cell or two) where $m=0$.

So $m$ of the initial problem is a sum of interior of subproblems that all are equal to zero. This proves the theorem for $k$ even.

If $k$ is an odd number, we just remark that graph of geodesics have no transversal edges, by definition. As every cell in such a $\Gamma(2 i+1, d)$ has a transversal edge, we remark that every cell in a graph of geodesic has size $2 k-2$. Removing transversal edges reduces the degree of vertices, but we note that for $k=3$ there are at most two transversal leaving edges for a vertex, and for $k>4$ there is at most one.

So the proof is the same for $\Gamma(3, d)$ as for $\Gamma(4, d-2)$, and the same for $\Gamma(2 j+5, d)$ as for $\Gamma(4 j+8, d-1)$. For $d=3$, we have to rearrange the initial proof but the result is the same.

Corollary 1. Let $x, y$ be two points of a $\Gamma(k, d)$ and $0<\delta<d(x, y)$. There are at most two points with distance $\delta$ from $x$ and distance $d(x, y)-\delta$ from $y$.

Hence, the set of geodesics between two points is a "narrow" graph (of a width of at most one cell). We must remark that, in every hyperbolic network $\Gamma(k, d)$ we can find two points as far from each other as we want such that there is only one unique geodesic between them and we can find, in the same way, two points with two disjoint geodesics.

\section{$5 \quad$ Triangle Inequality}

In this section, we not only show that the metric space of an hyperbolic network is hyperbolic (it is a known result), but give computable results about geometrical properties due to hyperbolic metric.

Theorem 3 (Theorem of void triangles). Let $x, y$ and $z$ be three points of an hyperbolic $\Gamma(k, d)$. Let the border (the triangle) be the cycle composed by three arbitrary geodesics $x y, y z$ and $z x$. Then, if the triangle has a minimal area (it means that it doesn't contain another xyz triangle in its interior), it does not contain any interior point for $d>3$ and at most one point for $d=3$. 


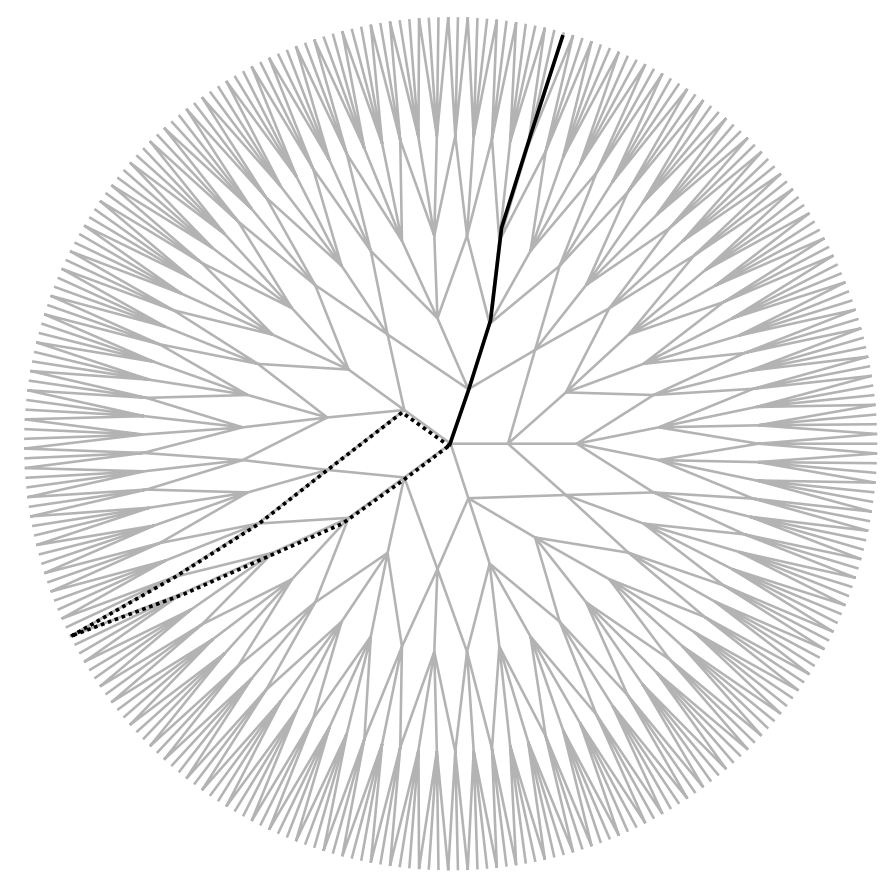

Fig. 6. Two types of geodesics

Proof. As the previous theorem, we suppose $m>0$ and we use an upper bound of edges on the graph of border $c=x y y z z x$. On each third of the border, we can remark the necessary presence of two leaving arcs every $k-2$ consecutive vertices, due to the fact that the considered path is a geodesic, and that we do not have another geodesic "inside".

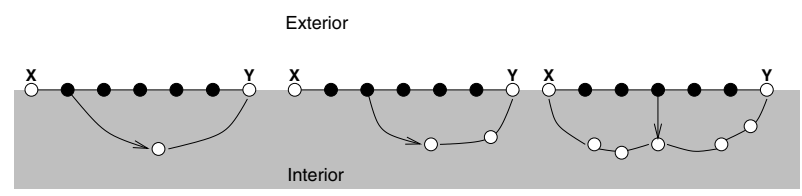

Fig. 7. Impossible to have only one leaving arc on $k-2$ vertices with the necessity of having only one geodesic between $\mathrm{X}$ and $\mathrm{Y}$ (for $k=7$ here)

So we have the following inequality :

$$
n+m-1+\frac{(n+2 m-2)}{(k-2)} \geq \frac{m d}{2}+n+\frac{n}{k-2}-\frac{3}{2}
$$

that we can simplify :

$$
(k-6) \geq m \gamma
$$


Hence if $d \geq 4$ we can verify that we have $m \leq 0$ for any hyperbolic $\Gamma(k, d)$. If $d=3$, we can verify that the case $m=1$ can be obtained in some little triangle (even minimal for $k$ odd) as we can see from figure 5 .

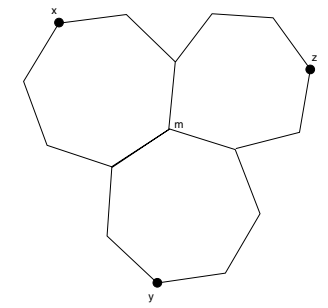

Fig. 8. A $x y z$ triangle with an interior point

Corollary 2. We note $D(x \mid y, z)$ the maximum distance from $x$ to a point in the intersection of the subgraphs of geodesics $x y$ and $x z$ (it is always defined as $x$ must be in this intersection). We consider $x^{\prime}$, such a point with distance $D(x \mid y, z)$ from $x$. If we look at the triangle $x^{\prime} y z$, we can deduce :

$$
D(x, z)+D(x, y) \geq D(y, z) \geq D(x, z)+D(x, y)-2 . D(x \mid y, z)-2 k
$$

We must note that $D\left(x^{\prime} \mid y, z\right)=0$ in that example. Hence, distance on a hyperbolic discrete network is working in a similar way as in trees.

Corollary 3. Due to the characterization of geodesics, we can remark that, if $d>3$ and $k>4$, every triangle (even non minimal) can not have any interior points.

This Theorem could be extended from triangles (3-gones) to $g$-gones. The result would be the same for $d \geq g$. It means that it is difficult to have a border with a large area. When $d$ is great, so is the curvature $\gamma$, and areas are delimited by very unsmooth large borders.

\section{Conclusion}

Now, we know the properties of a hyperbolic network : areas are small, perimeters very unsmooth and discrete geodesics are thin. So we can see such networks like a compromise between common Euclidean network, with quadratic areas, simple perimeter and a lots of geodesics; and infinite trees, with infinite areas and unicity of geodesics. 
For network algorithms, hyperbolic networks have the qualities of Euclidean grids, like good connectivity but as we can see from this article, they have qualities of tree networks, like efficient broadcast (exponential number of neighbors), and specific routing methods (thinness of geodesics).

For graphical purpose, the main problem is still in the representation of such networks. They "grow" very fast and are very difficult to show in an efficient way. Mathematicians use some finite nice way of representation that is completely useless for practical uses. The solution seems to implement some three dimensional representation using the underlying hyperbolic structure of the network. This could lead to a better understanding of every hyperbolic three dimensional surface, allowing best polygonalization of special shapes.

\section{References}

1. J. M. Chassery, A. Montanvert, Géométrie discrète en analyse d'images, Hermès, Paris (1991). 149

2. H. S. M. Coxeter, W. O. J. Moser, Generators and relations for discrete groups, Springer-Verlag, Berlin . Göttingen 1957. 150

3. E. Ghys, C. de la Harpe, Sur les groupes hyperboliques d'après Michaël Gromov, Birkhäuser Ed., (1990). 150

4. J. P. Reveillès, Géométrie discrète, calcul en nombre entiers et algorithmique, Thèse d'Etat, Université L. Pasteur, Strasbourg (1991). 149 